\title{
Melting of a repulsive screened Coulomb system in two dimensions: Effect of corrugation
}

\author{
Hyangsuk Seong, S. D. Mahanti, and Surajit Sen \\ Department of Physics and Astronomy and the Center for Fundamental Materials Research, \\ Michigan State University, East Lansing, Michigan 48824 \\ Tahir Çağin \\ Molecular Simulations Inc., 199 S. Robles Ave., Suite 540, Pasadena, California 91101 \\ and Beckman Institute, California Institute of Technology, Pasadena, California 91125
}

(Received 27 May 1992)

\begin{abstract}
By use of constant energy molecular dynamics simulations, we have investigated the melting and freezing transitions in a two-dimensional system consisting of a constant density of classical particles interacting with a repulsive screened Coulomb (Yukawa) potential. In particular, we have investigated the role of an incommensurate substrate corrugation potential of sixfold symmetry on these transitions by probing the temperature dependence of the bond orientational order parameter $\left(\psi_{6}\right)$ and the corresponding susceptibility $\left(\chi_{6}\right)$. Other physical quantities such as energy, diffusion constant, and the density of local topological defects have been monitored through the transition region. In the absence of a corrugation potential the system shows a sharp melting transition. In the presence of a corrugation potential the transition temperature increases and the transition becomes smoother. In contrast to the corrugationfree case we find a broad peak in $\chi_{6}$ above the transition temperature. We interpret this behavior in terms of the melting of a domain-wall solid. The detailed nature of this smooth transition depends on the degree of incommensurability. Relevance of these results to stage- 2 graphite intercalation compounds is discussed.
\end{abstract}

\section{INTRODUCTION}

Physical properties of two-dimensional (2D) solids and liquids and the nature of the solid-liquid transition continue to be of theoretical and experimental interest since the dislocation- and disclination-mediated melting ideas of Kosterlitz and Thouless, ${ }^{1}$ Nelson and Halperin, ${ }^{2}$ Young, ${ }^{3}$ and others. ${ }^{4-6}$ The nature of the melting transition (whether first-order driven by grain-boundary melting $^{7}$ or continuous driven by unbinding of above topological defects) and the existence of the intermediate "hexatic phase" with quasi-long-range positional order and long-range bond orientational order sandwiched between the "2D solid" phase and the "2D isotropic liquid" phase are still uncertain. ${ }^{4}$

In real physical systems such as atoms adsorbed on 2D substrates or intercalation compounds with sufficiently weak interlayer interaction that renders these systems quasi-two-dimensional, the dominant effect of the substrate is the periodic corrugation potential. The latter affects the structures of both the solid and the liquid phases and the solid-liquid phase transition. ${ }^{2,8,9}$ In particular, one has to take into consideration both the commensurability of the periodic potential and the strength of the corrugation. In the limit of weak corrugation, Nelson and Halperin ${ }^{2}$ have argued that for an incommensurate potential the spatial variation of the NovacoMcTague angle ${ }^{10}$ gives rise to a new elastic constant $(\gamma)$ in addition to the two usual Lamé constants $(\lambda$ and $\mu)$ associated with a $2 \mathrm{D}$ isotropic elastic solid. The elastic constant $\gamma$ determines the energy associated with the spatial variation of the bond angle field in the presence of a finite substrate potential. Thus the corrugation potential acts as a field which couples to the bond orientational order parameter. Consequently, one has only the dislocation unbinding transition, and the disclination unbinding transition is washed out just as a ferromagnetic transition gets washed out in the presence of a uniform magnetic field. For intermediate-to-strong incommensurate corrugation potential the precise nature of the transition is not known (see below the discussion in the lattice-gas limit).

When the corrugation potential is commensurate, the nature of the melting transition is profoundly affected. For coarse substrate mesh, the Kosterlitz-Thouless picture becomes inappropriate and even for a weak substrate potential a lattice-gas picture describes the solid-liquid phase transition. ${ }^{2}$ For sufficiently fine substrate corrugation, the commensurate solid undergoes a transition to a floating solid phase, which then melts through dislocation unbinding. In this case the disclination unbinding transition is also washed out. In the limit of extremely strong corrugation potential the system of course behaves like a lattice gas. ${ }^{11}$ Even in this case Nelson and Halperin speculated that one could have a melting process similar to that for a continuum model if the corrugation mesh size was sufficiently small. Thus one expects to see profoundly different melting behavior depending on the strength and the periodicity of the corrugation potential. If, on the other hand, the melting transition is determined by the grain-boundary condensation, ${ }^{7}$ one would like to know the effects of the corrugation on the grainboundary formation and hence on the transition.

Physical systems such as physisorbed rare-gas atoms on graphite, ${ }^{12} \mathrm{H}_{2}\left(\mathrm{D}_{2}\right)$ on graphite, ${ }^{13}$ and graphite intercalation compounds ${ }^{14}$ fall in the weak-to-intermediate corrugation strength limit. In these systems the wave- 
length of the corrugation potential $\left(\Lambda_{c}\right)$ is typically $r$ $(\sqrt{3} \leq r \leq \sqrt{7})$ times smaller compared to the systems' periodicity $\left(\Lambda_{0}\right)$ in the absence of corrugation. We do not know of any real physical system where $\Lambda_{c} \gg \Lambda_{0}$. Thus to understand the full scope of melting on a corrugated surface for different corrugation strengths and different substrate periodicities, it is necessary to undertake a systematic simulation study of the melting phenomena for systems with different densities and different strengths of the corrugation.

Recently, Vives and Lindgard ${ }^{15}$ have carried out extensive Monte Carlo simulations on a commensurate $(\sqrt{3} \times \sqrt{3})$ Lennard-Jones (LJ) system to study the nature of the transition and the decay of translational and bond orientational correlations as a function of the strength of corrugation. They ${ }^{16}$ have also extended these calculations to the incommensurate case appropriate for the $\mathrm{H}_{2}\left(\mathrm{D}_{2}\right)$-graphite system although the major emphasis in this work was to understand the low-temperature structure. Earlier, Abraham and his collaborators have carried out extensive simulation studies of the rare-gas atoms physisorbed on graphite. ${ }^{9}$ These simulation studies are on LJ systems. In this paper, we use moleculardynamics (MD) simulation to study the role of incommensurate corrugation potential for a 2D system of atoms interacting via a repulsive Yukawa potential, a realistic representation of intercalation compounds where the intercalants are charged objects. It should be noted here that screened Coulomb systems, such as the one addressed here, take significantly more computation time (due to the exponential form of the interaction) compared to the LJ and other (algebraic) "short-range" systems. Although we expect some general characteristics of Lennard-Jones and repulsive Yukawa systems to be similar, it is of interest to compare the detailed structure and the nature of the transition in these two potential systems. $^{17}$

The arrangement of the paper is as follows: In Sec. II we discuss briefly the physical systems under study, namely the graphite intercalation compounds and the nature of the potential. Specifically we discuss the case of higher-stage graphite intercalation compounds (GIC's) which have been extensively studied experimentally. In Sec. III we discuss our MD simulation method and the systems studied. The temperature dependence of different physical quantities is studied for different corrugation strengths in Sec. IV. In Sec. IV we also compare the results for two systems with slightly different densities; one of which shows a perfectly ordered periodic domain-wall structure (to be detailed in Sec. II, below) as its ground state. Finally, in Sec. V we summarize the important results of the present work and discuss its implications vis-ă-vis earlier work on LJ and related systems.

\section{THE PHYSICAL SYSTEM AND THE POTENTIAL MODEL}

The physical system that we study here is stage-2 alkali-metal GIC. ${ }^{18,19}$ In particular, we study the $\mathrm{RbC}_{24}$ GIC, the room-temperature (liquid phase) static and dynamic structure factors of which have been studied in considerable detail recently. ${ }^{19-23}$ Very good agreement between MD simulation results and experiment has been obtained using a simple two-body repulsive Yukawa potential between $\mathrm{Rb}$ ions and an external corrugation potential to describe the effect of the graphite substrate. The strength of the corrugation potential was obtained by fitting to the liquid state structure factor data. ${ }^{19}$ We have recently shown that the same potential can describe the low-temperature domain and domain-wall structure in this compound, which agrees very well with the $x$-raydiffraction results. ${ }^{18,21,22}$ Thus a $2 \mathrm{D}$ model of ions interacting via a short-range screened Coulomb potential in the presence of a corrugation potential provides an excellent description of the stage- $n(n \geq 2)$ graphite intercalation compounds.

The $\mathrm{Rb}$ ions are assumed to interact via the following two-body potential:

$$
V^{(2)}\left(\mathbf{r}_{1}, \mathbf{r}_{2}\right)=\frac{q^{2} \exp \left(-\Gamma\left|\mathbf{r}_{1}-\mathbf{r}_{2}\right|\right)}{\left|\mathbf{r}_{1}-\mathbf{r}_{2}\right|},
$$

where the charge on each $\mathrm{Rb}$ ion $q=4.8028 \times 10^{-10} \mathrm{esu}$ and the screening parameter $\Gamma=0.49 \AA^{-1}$. As mentioned before, the one-body graphite corrugation potential was obtained by Moss et al. ${ }^{19}$ by fitting to the liquid structure factor data and is given by

$$
\begin{aligned}
V^{(1)}(\mathbf{r})=2 K\{ & 2 \cos (2 \pi x / a) \cos (2 \pi y / \sqrt{3} a) \\
& +\cos (4 \pi y / \sqrt{3} a)\}
\end{aligned}
$$

with the constant $2 K=-0.9$ (in reduced units). In energy units $2 K / k_{B}=-270 \mathrm{~K}$. The constant $a=2.46 \AA$ is the distance between the centers of the nearest graphite hexagons. The above two-body potential was used by Chen, Karim, and Pettitt ${ }^{23}$ in their MD simulations. Moss et al. ${ }^{19}$ used a different form of the two-body potential in their MD simulation studies. In the region of interest the latter potential is weaker than the one used in the present simulation studies.

Our MD simulations have been performed with $216 \mathrm{Rb}$ ions distributed to ensure a planar density of 12 Carbon/ $\mathrm{Rb}$ (corresponding to the stage- $2 \mathrm{RbC}_{24}$ system), i.e., $0.0318 \mathrm{Rb}$ ions $/ \AA^{2}$. The cell edges are arranged to lie on the fixed corrugation minima and periodic boundary conditions are imposed. In addition to this planar density, we have also studied a 301-particle system with a slightly smaller $\mathrm{Rb}$ density $\left(0.0311 \mathrm{Rb}\right.$ ions $\left./ \AA^{2}\right)$ which corresponds to the stage- $2 \mathrm{RbC}_{24.57}$ system. The ground state of the latter system turns out to be a perfectly ordered periodic domain-wall (PDW) structure, ${ }^{22}$ where slightly distorted triangular plaquettes consisting of $7 \mathrm{Rb}$ ions (nano domains) are periodically arranged, separated by a periodic array of domain walls. In contrast, the ground state of $\mathrm{RbC}_{24}$ can be described by the above PDW structure with additional (about $2.2 \%$ more) $\mathrm{Rb}$ ions occupying interstitial sites. We will refer to these additional $\mathrm{Rb}$ atoms as atomic (distinct from topological) defects. The reason for choosing these two nearby densities is to see whether the melting process and related thermodynamic quantities are sensitive to these defects. In this paper, $\mathrm{RbC}_{24}$ with $2 K=0$, and $2 K=-0.9$ will be referred to as systems I and II, respectively, and $\mathrm{RbC}_{24.57}$ with $2 K=-0.9$ will be referred to as system III. 


\section{DETAILS OF MD SIMULATION}

The MD simulations were carried out using a sixthorder Gear predictor-corrector algorithm ${ }^{24}$ to solve the Newton's equations. The time step was taken to be 0.0029 picoseconds (ps) (or $0.0072 \mathrm{ps)}$ ) and typical equilibration time was at least 350 ps for the 216- and 301particle systems. The energy per ion was found to be conserved to $99.994 \%$ accuracy over 150 -ps run periods for both these systems. The range of the two-body potential was kept within a circle of radius $27.06 \AA$ centered on any $\mathrm{Rb}$. The results remained essentially the same when a larger cutoff radius was used. Most of the simulations were carried out in the temperature range $100<T<300$ $\mathrm{K}$ and in some cases we cooled down the system to as low as $3 \mathrm{~K}$. For system I whose ground state is a triangular lattice, we started the simulations from the hightemperature liquid phase, slowly cooled the system in temperature intervals of $10 \mathrm{~K}$ through the transition region down to $3 \mathrm{~K}$, and then heated the system through the transition to look for any hysteresis effect. System II was initially heated from an assumed zero temperature structure and allowed to melt and equilibrate. It was then slowly cooled through the transition region. Finally, the cooled solid was again slowly heated to see whether liquid-solid transition showed hysteresis. For the 301particle $\mathrm{RbC}_{24.57}$ system (system III) we chose a different initial condition in order not to bias our simulations toward a PDW structure ${ }^{22}$ as its ground state. For this system we chose an initial configuration where the positions of the $\mathrm{Rb}$ ions were randomly distributed; this corresponded to an extremely hot nonequilibrium system. The system cooled down rather quickly to $300 \mathrm{~K}$ from an initial nonequilibrium temperature of $\sim 10^{4} \mathrm{~K}$ and was allowed to equilibrate at that temperature for a sufficiently long time. We then slowly cooled the system by temperature intervals of $10 \mathrm{~K}$ until the ground state was reached and it turned out to be the expected PDW solid. The solid was then slowly heated to probe the solid-liquid transition. For the three systems, results of cooling and heating runs were compared to ensure that thermal equilibrium was achieved.

\section{RESULTS}

Before discussing the nature and the details of the melting transition, we present in Fig. 1 the structure factor $S(\mathbf{k})$ for the system II obtained at two different temperatures, $T=250 \mathrm{~K}$ corresponding to the liquid phase and $T=3 \mathrm{~K}$ corresponding to the solid phase. The liquid is clearly structured due to the presence of the corrugation potential as seen in the anisotropy of $S(\mathbf{k}) .^{19-23}$ The domain structure of the solid phase has been recently discussed by us $^{22}$ and agrees very well with the dominant features of the experimental structure factor. ${ }^{18}$ This gives us confidence in the adequacy of the potential we have used [Eqs. (2.1) and (2.2)] to describe the physical properties of the higher-stage Rb GIC's. In Fig. 2, we show the actual arrangement of the $\mathrm{Rb}$ ions in the solid phase for systems II and III. System II (stoichiometry $\mathbf{R b C}_{24}$ ) shows an imperfect domain structure [Fig. 2(a)], whereas

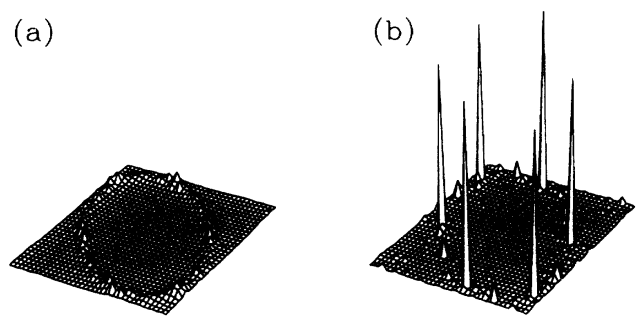

FIG. 1. Structure factor $S(\mathbf{k})$ for $\mathrm{RbC}_{24}$ (a) in the liquid and (b) in the solid phase obtained from MD simulation of a 216particle system (system II, see text). The liquid structure factor at $T=250 \mathrm{~K}$ shows an anisotropic ridge due to the effect of substrate corrugation. The solid structure factor has sharp peaks and only the dominant peaks $I_{1}$ and $I_{2}$ (see Ref. 22) and their sixfold symmetric counterparts are clearly seen.

system III (stoichiometry $\mathrm{RbC}_{24.57}$ ) shows a perfectly ordered PDW structure [Fig. 2(b)], i.e., the ground state is a commensurate structure. Note, however, that only the atoms at the center of the $\sqrt{7} \times \sqrt{7}$ domains sit at the minima of the single-particle potential, whereas the other atoms are displaced from these minima, but very slightly. The net effect of this displacement on the static structure factor $S(\mathbf{k})$ is a small change in the intensities of the main diffraction peaks. The PDW systems can be used to systematically study the thermodynamic properties of solids where the size of the domains and the domain walls are comparable. ${ }^{25}$

We next discuss the melting of systems II and III and compare their melting process with that of a corrugation-free system (system I), the usual triangular

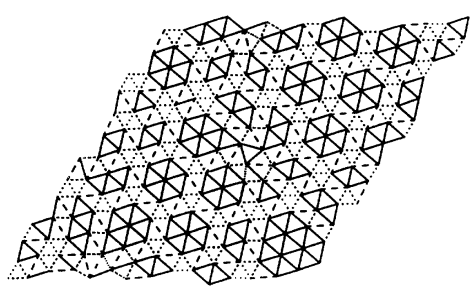

(a)

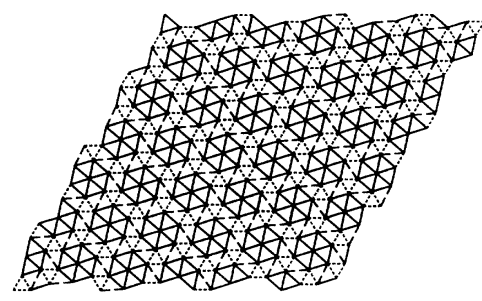

(b)

FIG. 2. (a) Real-space configuration at $T=3 \mathrm{~K}$ for (a) $\mathrm{RbC}_{24}$ (system II) and (b) $\mathrm{RbC}_{24.57}$ (system III). Domains and domain walls are clearly seen. The full, dotted, and the dashed lines correspond to the nearest-neighbor distances which are close to $\sqrt{7}, 2$, and 3 (in units of the graphite lattice constant), respectively. 
solid. In Fig. 3, we give the temperature dependence of the potential energy (total energy $-k_{B} T$ ) per particle as a function of $T$ both for the heating and the cooling runs. In the absence of the corrugation potential $(K=0)$, there is a rapid change in this energy in the temperature range $195<T<200 \mathrm{~K}$. The transition is most likely first order as seen in previous MD simulation of other physical systems [Lennard-Jones (LJ), ${ }^{9,26} 1 / r^{n}$ systems, ${ }^{27} e^{-\Gamma r} / r$ system, ${ }^{28}$ and the Weeks-Chandler-Anderson (WCA) system, which is a truncated version of the $\left.\mathrm{LJ}_{\text {system }}{ }^{29}\right]$. The absence of hysteresis might be due to either the small size of our systems or weak first-order nature of the transition. We note here that the earlier MD simulation studies of a repulsive screened Coulomb system were carried out for smaller (100 particles) systems in the absence of corrugation and showed hysteresis effects. ${ }^{28}$ The details of the equilibration times at different temperatures in this study are not available, but it is possible that the observation of hysteresis might be the result of nonequilibrium effects.

If we assume that system I shows a first-order transition and take the slopes of the energy vs $T$ above $210 \mathrm{~K}$ and below $190 \mathrm{~K}$, then we find the change in entropy associated with this first-order transition to be $\Delta s / k_{B}=0.23$, which agrees rather well with the earlier simulation results on other systems. ${ }^{27}$ As seen in Fig. 3, the effect of corrugation is drastic; in contrast to the corrugation-free case (system I), the potential energy changes smoothly for both systems II and III in the tem-

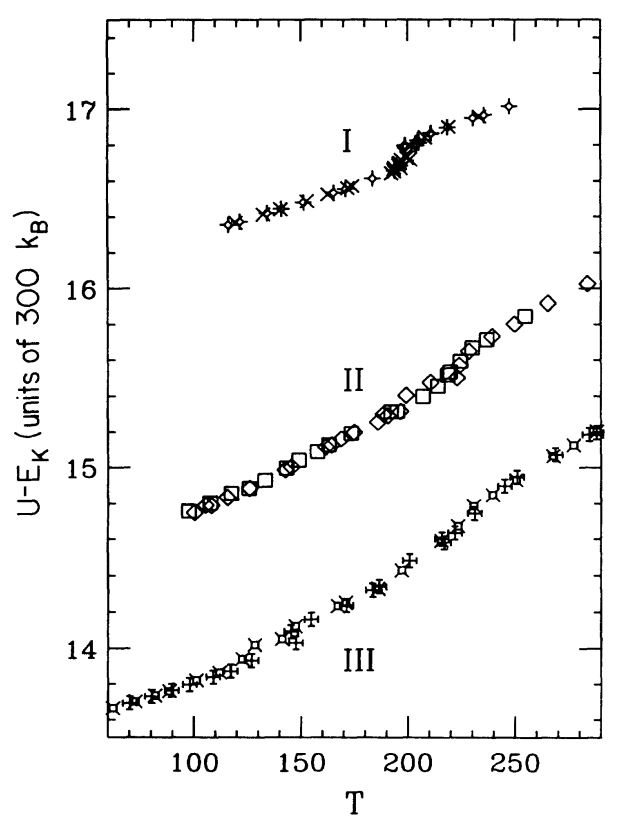

FIG. 3. Potential energy vs temperature with and without corrugation potential. Energy is measured in units of $300 k_{B}$ and the temperature in units of Kelvin. System I corresponds to $\mathrm{RbC}_{24}$ without corrugation $(2 K=0)$, system II corresponds to $\mathrm{RbC}_{24}$ with corrugation $(2 K=-0.9)$, and system III corresponds to $\mathrm{RbC}_{24.57}$ with corrugation $(2 K=-0.9)$. $2 K$ is a measure of the strength of the corrugation potential [see Eq. (2.2)]. For all these three systems both cooling and heating results are shown. perature range $190<T<230 \mathrm{~K}$, although there is some indication of a slope change near about $220 \mathrm{~K}$. To make sure whether there is actually a solid-liquid phase transition in systems II and III, we must calculate other physical quantities which are sensitive to this transition. In addition, one must increase the strength of the corrugation systematically to see how the corrugation alters the nature of the melting transition. We have carried out some preliminary studies in this direction by choosing $2 K=-0.15$ and -0.36 . The details of these simulations will be discussed in a separate publication.

Since it is difficult to investigate the melting transition by calculating the positional correlation function for small systems, one usually monitors the temperature dependence of the bond orientational order parameter (BOOP) and its associated susceptibility. Strandburg, Zollweg, and Chester ${ }^{30}$ introduced the BOOP by defining a quantity $\psi_{6}$ given by

$$
\psi_{6}=(1 / N)\left|\sum_{l j}\left(1 / n_{l}\right) e^{i 6 \theta_{l j}}\right|,
$$

where the sum on $l$ is over all the $N$ particles, and the sum on $j$ is over the nearest neighbors of a particular particle $l ; n_{l}$ being the number of such nearest neighbors defined by the standard Voronoi construction. The angle $\theta_{l j}$ is the orientation of the bond joining the particles $l$ and $j$ measured with respect to a given fixed axis. In Fig. 4 we plot $\psi_{6}$ as a function of $T$ for systems I and II. In the corrugation-free case there is a rapid decrease in the order parameter between 190 and $200 \mathrm{~K}$, whereas in the presence of corrugation the drop in the order parameter appears to be less abrupt and the transition temperature is somewhere between 210 and $230 \mathrm{~K}$. In addition, in the solid phase the order parameter is larger in the corrugation-free case. This can be easily understood by looking at the structure in the solid phase. The system without corrugation has a perfect triangular lattice structure at $T=0 \mathrm{~K}$ for which $\psi_{6}=1$, whereas in the system with corrugation the domain walls reduce the strength of this order parameter. The nonzero value of the order pa-

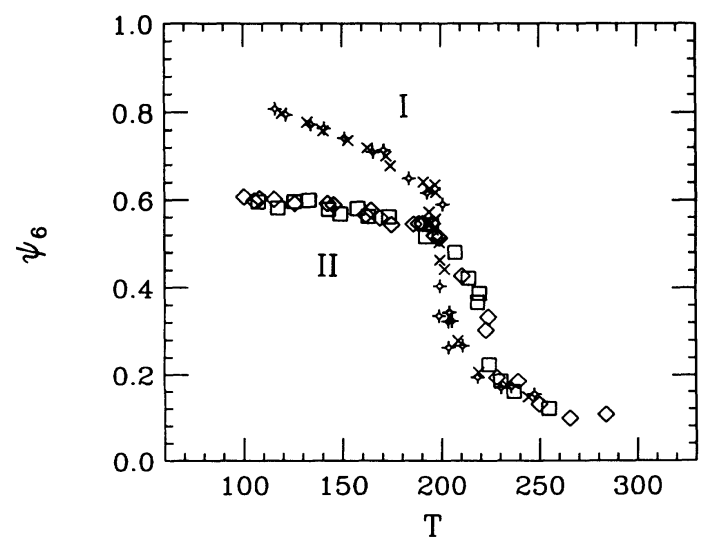

FIG. 4. Bond orientational order parameter $\psi_{6}$ as a function of temperature for systems I and II (see Fig. 3 caption for explanations). Both heating and cooling run results are shown. 
rameter in the liquid phase of system $I$ is due to finite-size and periodic boundary condition effects because $\psi_{6}$ should vanish in the thermodynamic limit. In the presence of the corrugation potential of hexagonal symmetry $\psi_{6}$ should be nonzero even in the thermodynamic limit. We note, however, that in our finite system simulations (with periodic boundary conditions), for temperatures above $240 \mathrm{~K}$ the residual order parameter is independent of the strength of the corrugation, suggesting that the influence of finite-size effects in the calculation of $\psi_{6}$ are indeed large.

To further probe the nature of the transition and to obtain the transition temperature more accurately, we have calculated the susceptibility associated with the BOOP. We define the angular susceptibility $\chi_{6}$ as

$$
\chi_{6}=N\left[\left\langle\left|\psi_{6}\right|^{2}\right\rangle-\left\langle\left|\psi_{6}\right|\right\rangle^{2}\right] / k_{B} T .
$$

In Fig. 5, we give the temperature dependence of $\chi_{6}$ for the two systems discussed in the previous paragraph. In the corrugation-free system, the susceptibility increases rapidly with decreasing $T$ and shows a dramatic drop (almost a discontinuous jump) between 200 and $195 \mathrm{~K}$. This is the same temperature range where the order parameter shows a rapid change (Fig. 4), thereby suggesting that the transition is most likely first order. From our finite-size simulation we cannot conclusively state that the transition is indeed first order. In the absence of an observable hysteresis we cannot rule out a sharp but continuous transition. Glasser and Clark, ${ }^{29}$ in their extensive MD simulation studies of a truncated $\mathrm{LJ}$ system, found a nearly discontinuous drop in $\chi_{6}$ as a function of increasing density (temperature being held constant) indicating a first-order transition. They did not, however, discuss the hysteresis issue.

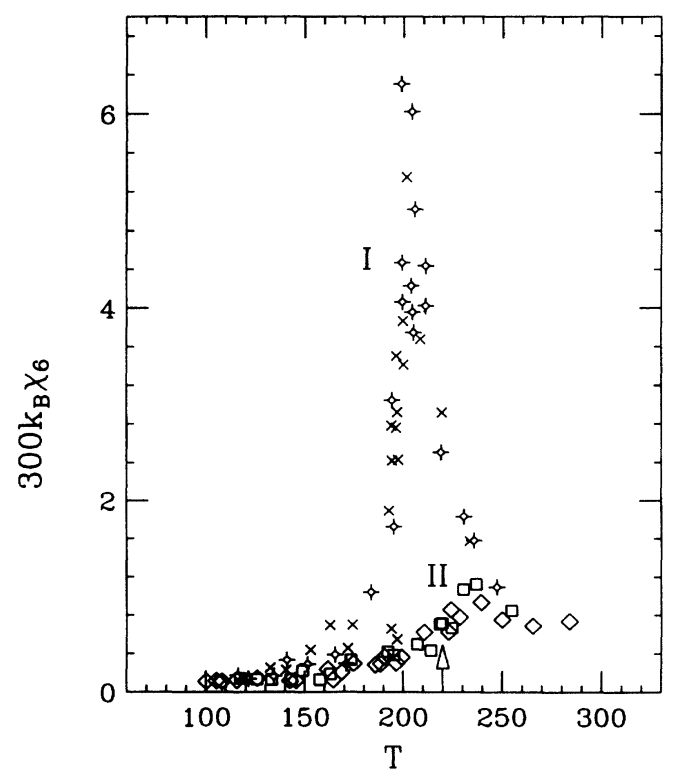

FIG. 5. Angular susceptibility $\chi_{6}$ [see Eq. (4.2) for the definition] vs temperature for systems I and II (see Fig. 3 caption for explanation). Heating and cooling run results are shown.
In the presence of corrugation the behavior of the susceptibility is much more intriguing. For the $\mathrm{RbC}_{24}$ system (system II), $\chi_{6}$ is relatively small and is a smooth function of temperature showing a very broad peak near 230-240 K. There is not much action near the temperature where the order parameter shows a rapid drop at $200<T<220 \mathrm{~K}$ (indicated by an arrow). Clearly the melting transition of this domain-wall solid is very different from that of a simple triangular solid (system I). In order to pin down the precise nature of the transition we are planning to carry out extensive Monte Carlo simulations in larger systems $(N=864)$ covering the transition region and also the region where the susceptibility shows a peak.

One plausible physical argument we can give to explain the temperature dependence of $\psi_{6}$ and $\chi_{6}$ in the domainwall solid case is the following: ${ }^{31}$ As we increase the temperature, the region near the domain walls starts to soften or "melt" (near $\sim 200 \mathrm{~K}$ ); this leads to a rapid decrease in the long-range bond orientational order. However, above this transition there is still sufficient shortrange orientational correlation inside each triangular domain and the peak in $\chi_{6}$ can be thought of as a second "melting" of these highly correlated small triangular lattice clusters of $\sqrt{7} \times \sqrt{7}$ domains, the transition region being broadened by finite-size effects. The fact that this broad peak is seen at temperature $\sim 240 \mathrm{~K}$, which is higher than $200 \mathrm{~K}$ where the corrugation-free $\sqrt{6} \times \sqrt{6}$ triangular solid melts, can be understood by the following simple argument. Ordinarily a $\sqrt{7} \times \sqrt{7}$ triangular solid in the absence of corrugation will melt at a temperature lower than $200 \mathrm{~K}$. However, in the presence of a strong corrugation potential the melting temperature will be increased from this value and it is possible that the melting temperature will be near $240 \mathrm{~K}$ where one sees the broad peak in $\chi_{6}$. In fact, Vives and Lindgard, ${ }^{15}$ in their MC simulation study of a commensurate $\mathrm{LJ}$ system, found an increase in the melting transition temperature with an increase in the strength of the corrugation.

Since the ground state of $\mathrm{RbC}_{24}$ can be described by a perfectly ordered PDW solid with a few additional $R b$ atoms (defects) and since it is reasonable to expect that the order parameter, the susceptibility, and the nature of the transition might be affected by these defects, we have studied the temperature dependence of $\psi_{6}$ and $\chi_{6}$ for the stage-2 $\mathrm{RbC}_{24.57}$ (system III) whose ground-state structure is shown in Fig. 2(b). In fact, one can study the melting properties of a whole class of PDW solids where the widths of the domains and domain walls can be systematically varied by simply changing the planar $\mathrm{Rb}$ concentration. ${ }^{22}$ This will tell us how the nature of the melting transition changes as we introduce domain walls into the system. For $\mathbf{R b C}_{24.57}$ where the $\sqrt{7} \times \sqrt{7}$ triangular domains contain seven atoms separated by $2 \times 3 \times \sqrt{7}$ walls, the order parameter at low temperatures is slightly larger than $\mathrm{RbC}_{24}$ [see Fig. 6(a)] consistent with the presence of additional defect atoms in the latter system. For the former we see a phase transition (rapid decrease of $\psi_{6}$ ) at $T=230 \mathrm{~K}$ [Fig. 6(a)] with a peak in the susceptibility $\chi_{6}$ [see Fig. 6(b)] at a higher temperature $(T=240 \mathrm{~K})$. This is qualitatively similar to that shown by system II. 


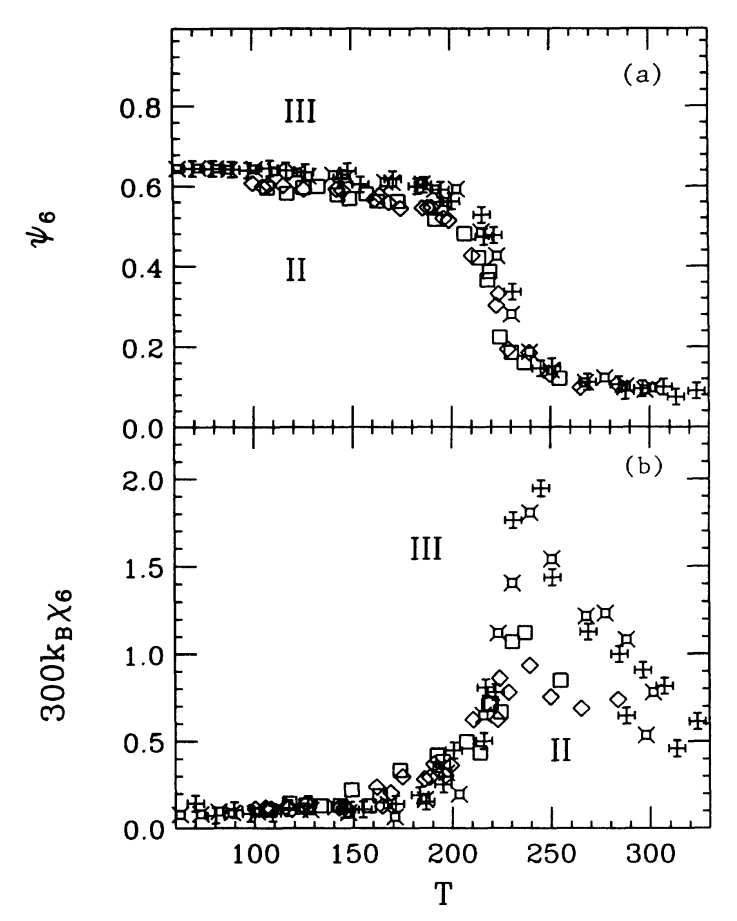

FIG. 6. Temperature dependence of (a) bond orientational order parameter $\psi_{6}$ for systems II and III and (b) corresponding angular susceptibility $\chi_{6}$. See Fig. 3 caption for the description of different systems. Both heating and cooling runs are shown.

Thus we conclude that the peak in orientational susceptibility above the melting transition appears to be a general property of the melting of domain-wall solids. The removal of defects makes the susceptibility peak in III slightly more pronounced compared to the system II. But in both cases the peak intensity is about 3-5 times smaller than the corrugation-free case.

To further explore the effect of corrugation on melting we have studied the temperature dependence of the concentration of local topological defects (LTD's), which are bound pairs and quartets of five- and sevenfold coordinated atoms both in the presence and the absence of corrugation. We use the standard Voronoi polyhedra construction to obtain the number of five-, six-, and sevenfold coordinated atoms. It appears that the effect of corrugation on the density of these LTD's is marginal excepting in the transition region. In the transition region, the temperature dependence of the defect density $\rho_{d}$ is much smoother for the corrugation case (see Fig. 7), indicating a continuous transition. One interesting observation is that in the solid phase $(T<195 \mathrm{~K})$ and in the liquid phase $(T>250 \mathrm{~K}) \rho_{d}$ is weakly dependent on the corrugation strength $(2 K)$. The corrugation potential has a strong effect on the defect density only in the transition region. When $2 K=0$, we see that the rate of increase of $\rho_{d}$ with temperature, $d \rho_{d} / d T$, is maximum at $T=200 \mathrm{~K}$, which is also the maximum of $\chi_{6}$. In contrast, for $2 K=-0.9$, i.e., for the system II, $d \rho_{d} / d T$ is maximum near $T=220 \mathrm{~K}$, whereas $\chi_{6}$ peaks at $T \sim 240 \mathrm{~K}$, again indicating a basic difference between the melting characteristics of a triangular solid in the absence of corruga-

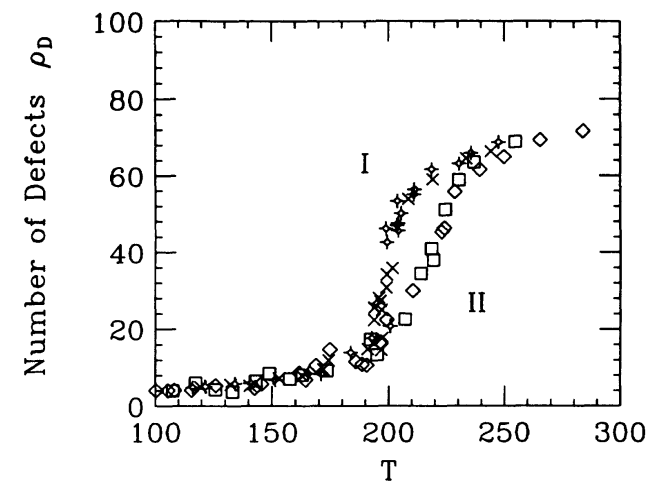

FIG. 7. Number of disclinations (topological defects, fiveand sevenfold coordinated atoms) vs temperature. Observe the sharp increase in the number of disclinations near $200 \mathrm{~K}$ for system I (without corrugation) and smoother increase near 220 $\mathrm{K}$ for the system II (with corrugation). Different symbols refer to heating and cooling runs.

tion and a domain-wall solid.

Finally, the translational diffusion constant $D$ has been calculated to monitor the solid-liquid transition and the effect of the corrugation on the diffusion rate. In contrast to its effect on the density of topological defects, corrugation profoundly affects the diffusion rate (see Fig. 8). The nearly first-order nature of the transition in the corrugation-free case is seen as a dramatic increase in $D$ at about $200 \mathrm{~K}$. On the other hand, in the presence of corrugation $D$ starts to increase rather slowly near the transition temperature $(T=220 \mathrm{~K})$, seems to flatten slightly near $240 \mathrm{~K}$ where there is a peak in the orientational susceptibility, and finally increases linearly with $T$. The drastic reduction in $D$ in the liquid phase in the presence of corrugation can be understood in terms of an additional activation process involving the corrugation potential. Using the parameters of our potential we estimate the activation energy barrier associated with the

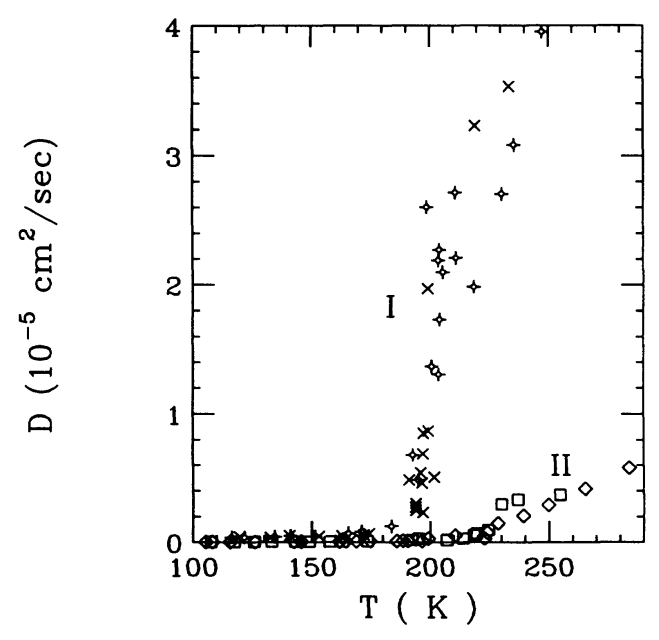

FIG. 8. Diffusion constant $D$ vs temperature for system I (without corrugation) and system II (with corrugation). Both heating and cooling runs are shown. 
corrugation potential to be about $1080 \mathrm{~K}$. The experimental value of the activation energy obtained from the diffusion measurements ${ }^{20}$ is $\sim 0.063 \mathrm{eV}(756 \mathrm{~K})$ which is about a factor $\frac{2}{3}$ of the single-particle activation barrier. The net activation energy is, of course, a combination of the single-particle and interaction potential. More detailed temperature-dependent study of the diffusion rate $D$ in the liquid phase is required to quantitatively determine the true activation energy. Further details of the diffusion rate and the nature of the dynamics will be discussed in a separate paper.

\section{SUMMARY AND DISCUSSION}

In summary, we have carried out extensive MD simulations of the melting and freezing transitions of a 2D repulsive Yukawa system in the presence of (strong) incommensurate corrugation potential. This model describes rather well the physical properties of stage $n$ $(n \geq 2)$ graphite intercalation compounds and similar systems where the intercalants are charged objects interacting with a screened Coulomb interaction. The parameters we have chosen are appropriate for stage-2 Rb GIC. Before comparing our simulation results with experiments in Rb stage-2 GIC we summarize some of our general observations.

The triangular lattice structure of the corrugation-free solid changes to a periodic domain-wall structure in the presence of a (strong) incommensurate potential. The size of the domains and of domain walls depend on the strength of the corrugation potential and the intercalant density. Whereas the former (triangular solid) undergoes a sharp (perhaps discontinuous) melting-freezing transition, the latter (PDW solid) shows a smooth transition. The transition temperature increases in the presence of corrugation. Such an observation was made by Vives and Lindgard ${ }^{15}$ in their Monte Carlo study of the melting of $\sqrt{3} \times \sqrt{3}$ commensurate $\mathrm{LJ}$ systems. In the absence of corrugation, we find that the rapid decrease in the bond orientational order parameter $\psi_{6}$ and the peak in the corresponding susceptibility $\chi_{6}$ occur at the same temperature. In contrast, in the presence of the corrugation potential, $\chi_{6}$ does not show any structure (discontinuity of peak) at the temperature where $\psi_{6}$ shows a rapid decrease, but a rather broad weak peak appears several degrees above this temperature. We have given a physical explanation of this behavior in terms of the melting of a domain-wall solid.

The temperature dependence of $\chi_{6}$ for the corrugation-free system shows a sharp $\lambda$-like peak. The ratio of the half width $\Delta T$ to the transition temperature
$T_{c}$, i.e., $\Delta T / T_{c} \approx 0.1$. Recently Glasser and Clark ${ }^{29}$ have carried out extensive MD simulations on a truncated LJ system (WCA system) and have studied the melting transition at a constant $T$ but as function of the density. They also find a sharp change (actually a discontinuity) in $\chi_{6}$ near the melting transition indicating a discontinuous transition, very much like what we have seen as a function of temperature at constant density. The half width of the peak in $\chi_{6}$ vs $\rho, \Delta \rho / \rho_{c}=0.22$. In contrast to our $M D$ results, the MC results (for $L J$ systems) of Vives and Lindgard ${ }^{15}$ in larger systems show an extremely broad peak in $\chi_{6}$ and appreciable hysteresis, most likely due to nonequilibrium effects. Although our system size is small, our results are for well-equilibrated systems as indicated by near agreement of the heating and cooling runs. Therefore we are unable to compare the sharpness of the susceptibility peak in the corrugation-free limit for repulsive Yukawa and $L J$ systems.

Finally, we would like to compare our simulation results with available experiments. It is not possible to directly measure the bond orientational order parameter and the associated susceptibility. The former can be indirectly measured from the anisotropy of the x-ray (or neutron)-diffraction peaks in the "solid phase." Zabel and co-workers ${ }^{20}$ measured the intensity of the superlattice peak as a function of temperature using neutron diffraction. They find a continuous transition from the solid to the liquid phase at about $165 \mathrm{~K}$ in stage- $2 \mathrm{RbC}_{24}$. Our simulation results agree with this excepting that our transition temperature is about $220 \mathrm{~K}$. This is due to (1) finite-size effect and (2) limitations in the potential model that we have used. In fact, Moss and co-workers ${ }^{19}$ have used a different two-body potential (but the same onebody potential as used here) and find the transition temperature to be somewhere near $160 \mathrm{~K}$. By comparing the two different two-body potentials in the region of physical interest we find that the Moss et al. potential is slightly weaker than ours. This can explain the difference in the transition temperature. However, we should point out that these authors did not probe the solid-liquid phase transition in any detail.

\section{ACKNOWLEDGMENTS}

This work has been supported in part by NSF DMR Grant Nos. 89-03579 and 90-24955 and by the Center for Fundamental Materials Research at Michigan State University. The authors acknowledge helpful discussions with Dr. W. Steele, Dr. M. L. Klein, Dr. K. Strandburg, and Dr. C. Jayaprakash.
1J. M. Kosterlitz and D. J. Thouless, in Progress in Low Temperature Physics, edited by D. F. Brewer (North-Holland, Amsterdam, 1978), Vol. VII-B, p. 373.

${ }^{2}$ D. R. Nelson and B. I. Halperin, Phys. Rev. B 19, 2457 (1979).

${ }^{3}$ A. P. Young, Phys. Rev. B 19, 1855 (1979).

${ }^{4}$ K. J. Strandburg, Rev. Mod. Phys. 60, 161 (1988).

${ }^{5}$ R. J. Birgeneau and P. M. Horn, Science 232, 329 (1986); N.
Grieser et al., Phys. Rev. Lett. 59, 1706 (1987).

${ }^{6}$ Q. M. Zhang and J. Z. Larese, Phys. Rev. B 43, 938 (1991).

${ }^{7}$ S. T. Chui, Phys. Rev. Lett. 48, 933 (1982).

${ }^{8}$ W. A. Steele (private communication).

${ }^{9}$ F. F. Abraham, Adv. Phys. 35, 1 (1986); Phys. Rev. B 28, 7338 (1983); 29, 2824 (1984).

${ }^{10}$ A. D. Novaco and J. P. McTague, Phys. Rev. Lett. 38, 1286 
(1977).

${ }^{11}$ L. K. Runnels, in Phase Transitions and Critical Phenomena, edited by C. Domb and M. S. Green (Academic, New York, 1972), Vol. 2.

${ }^{12}$ M. J. Colella and R. M. Suter, Phys. Rev. B 34, 2052 (1986); K. L. D'Amico, J. Bohr, D. E. Moncton, and D. Gibbs, ibid. 41, 4368 (1990); Q. M. Zhang and J. Z. Larese, ibid. 43, 938 (1991).

13J. Cui and S. C. Fain, Jr., Phys. Rev. B 39, 8628 (1989).

${ }^{14}$ S. C. Moss and R. Moret, in Graphite Intercalation Compounds: Structure and Dynamics, edited by $\mathrm{H}$. Zabel and S. A. Solin, Topics in Current Physics, Vol. 1 (Springer, New York, 1990).

${ }^{15}$ E. Vives and P. A. Lindgard, Phys. Rev. B 44, 1318 (1991).

${ }^{16} \mathrm{E}$. Vives and Per-Anker Lindgard (private communication).

${ }^{17}$ Recently structural phase transition (from bcc to fcc) and melting transition for a three-dimensional repulsive Yukawa system has been studied extensively by M. O. Robbins, K. Kremer, and G. S. Grest, J. Chem. Phys. 88, 3286 (1988).

${ }^{18}$ R. Clarke, J. N. Gray, H. Homma, and M. J. Winokur, Phys. Rev. Lett. 47, 1407 (1981); M. J. Winokur and R. Clarke, ibid. 54, 811 (1985); Phys. Rev. 34, 4948 (1986); see also M. J. Winokur, Ph.D. thesis, University of Michigan, 1986, where periodic arrangement of $\sqrt{7} \times \sqrt{7}$ domains was suggested for $\mathrm{RbC}_{24}$.

${ }^{19}$ S. C. Moss, G. Reiter, J. L. Robertson, C. Thompson, J. D. Fan, and K. Oshima, Phys. Rev. Lett. 57, 3191 (1986); J. D. Fan et al., Phys. Rev. B 39, 6111 (1989).

${ }^{20}$ H. Zabel, A. Magerl, J. J. Rush, and M. E. Misenheimer, Phys. Rev. B 40, 7616 (1989); J. D. Fan et al., Phys. Rev. Lett. 64, 188 (1990).

${ }^{21}$ S. Sen et al., Recent Developments in Computer Studies in Condensed Matter Physics IV, edited by D. P. Landau et al. (Springer, Berlin, in press).

${ }^{22}$ Hyangsuk Seong, S. Sen, T. Çağin, and S. D. Mahanti, Phys.
Rev. B 45, 8841 (1992).

${ }^{23}$ Z. Chen, O. A. Karim, and B. M. Pettitt, J. Chem. Phys. 89, 1042 (1988).

${ }^{24}$ M. P. Allen and D. J. Tildesley, Computer Simulations in Liquids (Claredon, Oxford, 1987).

${ }^{25}$ The structure of the PDW solid is quite similar to the one proposed by J. Cui and S. C. Fain, Jr. (see Ref. 13) for the $\gamma$ phase of $D_{2}$ /graphite where $\sqrt{3} \times \sqrt{3}$ domains and $\sqrt{3} \times 1$ walls are periodically arranged. One should also note that all the $D_{2}$ molecules do not sit at the hexagon centers as in the PDW model (see Ref. 16).

${ }^{26}$ C. Udink and J. van der Elsken, Phys. Rev. B 35, 279 (1987).

${ }^{27}$ P. Vashista and R. K. Kalia, in Melting, Localization, and Chaos, edited by R. K. Kalia and P. Vashishta (NorthHolland, New York, 1982), p. 43.

${ }^{28}$ H. Chen, P. Dutta, D. E. Ellis, and R. Kalia, J. Chem. Phys. 85, 2232 (1986).

${ }^{29}$ M. Glasser, Ph.D. thesis, University of Colorado, 1991.

${ }^{30}$ K. J. Strandburg, J. A. Zollweg, and G. V. Chester, Phys. Rev. B 30, 2755 (1984).

${ }^{31}$ There is an alternate way of looking at the melting transition (suggested to us by Professor C. Jayaprakash), i.e., in terms of condensation of defects which are in these cases domain walls. One can visualize preexisting elemental $\sqrt{7} \times \sqrt{7}$ triangular plaquettes in the liquid phase above the solid-liquid transition. The region between these placquettes (domain walls) can be thought of as defects which then order below a critical temperature. Since, for the system size we have studied, the effective density of these defects is small (by about a factor of 7) compared to that of atoms, one sees a rather broad transition region. To test this interesting idea one has to study the melting of the PDW solid for larger systems and see if the transition region sharpens with the size of the system. 